\title{
0 Registro Civil nos quatro primeiros meses da pandemia de Covid-19 no Brasil: estudo comparativo entre óbitos por mês de ocorrência e de registro
}

\author{
Claudia Cristina de Aguiar Pereira* \\ Amanda Batista Marcelino** \\ Carla Jorge Machado ${ }^{\star \star \star}$
}

\begin{abstract}
O Registro Civil fornece informações aos estudos demográficos sobre mortalidade, fecundidade e nupcialidade. Questões têm surgido sobre o registro dos óbitos quando as causas de morte decorrem da Covid-19. A notificação pode acontecer com atraso. Com base nas informações disponibilizadas pelo Registro Civil de óbitos pela Covid-19, discriminados por dois grupos data de registro e data de ocorrência -, o presente estudo compara estes grupos por categoria de município (capital, região metropolitana e interior), no período de março a junho de 2020 , que corresponde aos quatro primeiros meses da pandemia no Brasil. Avaliam-se a magnitude e o sentido das diferenças de registros de óbitos entre os dois grupos. Foram utilizados gráficos e análise de regressão linear para comparações. Os achados indicam comportamento diferente de óbitos por mês de registro em comparação àqueles por mês de ocorrência entre março e junho. É importante identificar tal diferença de comportamentos dado que, para o monitoramento em curtíssimo prazo da pandemia, óbitos por data de registro antecipam óbitos cujo registro ainda será corretamente disponibilizado por data de ocorrência. Conclui-se que essa variação deve-se, principalmente, à correção e atualização sistemática das informações. Quanto à categoria de município, a maior diferença entre óbitos por mês de ocorrência e de registro foi observada para os municípios do interior, em consonância com o alastramento da epidemia rumo ao interior no mês de maio.
\end{abstract}

Palavras-chave: Infecções por coronavírus. Mortalidade. Sistemas de registros.

\footnotetext{
* Escola Nacional de Saúde Pública Sergio Arouca (Ensp) da Fundação Oswaldo Cruz (Fiocruz), Rio de Janeiro-RJ, Brasil (pereirac.claudia@gmail.com; https://orcid.org/0000-0003-1389-9214).

** Universidade Federal de Minas Gerais (UFMG), Belo Horizonte-MG, Brasil (dudamarcelino@gmail.com; https://orcid. org/0000-0001-9636-1086).

*** Universidade Federal de Minas Gerais (UFMG), Belo Horizonte-MG, Brasil (carlajmachado@gmail.com; https://orcid. org/0000-0002-6871-0709).
} 


\section{Introdução}

O Registro Civil é definido pelas Nações Unidas como o registro universal, compulsório, contínuo, permanente e confidencial das ocorrências dos eventos vitais dos indivíduos (UNITED NATIONS, 2020), como nascimento, morte, casamentos e separações, estabelecendo a existência legal de uma pessoa. Trata-se de fonte essencial para a produção das Estatísticas Vitais e está entre as atribuições fundamentais do poder público (MACHADO, 2020). As mortes registradas em cartórios em todo o país compõem o sistema de estatísticas do Registro Civil, coordenado pelo Instituto Brasileiro de Geografia e Estatística (IBGE). Este sistema, baseado em dados de cartórios, não tem a função de monitorar eventos vitais que ocorrem no território nacional, mas contribui para a detecção oportuna, pelos serviços de saúde municipais, estaduais e federais, de ocorrências atípicas nas várias localidades do país (FRANÇA et al., 2020). Assim, o Registro Civil fornece informações necessárias aos estudos demográficos sobre mortalidade, fecundidade e nupcialidade (IBGE, 2020). Usualmente, o registro do óbito ocorre a partir da apresentação da via amarela da Declaração de Óbito (DO), por um indivíduo que tenha o dever legal de fazê-lo junto ao cartório do Oficial de Registro Civil das Pessoas Naturais (CHOSSANI, 2020). 0 documento indica a causa básica da morte, sendo a base do sistema de informações sobre mortalidade.

Questões têm emergido quanto ao registro dos óbitos para causas de morte decorrentes da Covid-19. Algumas declarações de óbitos vêm sendo emitidas sem a confirmação prévia do diagnóstico viral para a Covid-19, em função do número insuficiente de testes laboratoriais para comprovar a doença (CHOSSANI, 2020). As dificuldades na identificação dos casos de Covid-19 surgem ainda de uma grande proporção de falsos negativos quando o teste é realizado, por diversos motivos (XIE et al., 2020). Além disso, mesmo quando submetidos a testes de alta acurácia, inúmeros indivíduos evoluem para óbito antes de terem seus resultados divulgados. Portanto, a inclusão oportuna de casos confirmados de Covid-19 no Sistema de Informação sobre Mortalidade (SIM) pode ser difícil e demorada (FRANÇA et al., 2020). Isso ocorre mesmo diante da recomendação aos profissionais médicos de indicar na DO quando houver suspeita de que o óbito tenha sido causado por doença respiratória em decorrência da infecção pelo vírus Sars-CoV-2.

Dessa forma, a notificação pode ocorrer com dias e até semanas de atraso, após a devida confirmação (FERNANDES, 2020). É possível, assim, que haja mudança na causa do óbito, o que se reflete no próprio Registro Civil e, portanto, nas estatísticas do Registro Civil: “Uma vez lavrado o óbito perante o cartório, e confirmado posteriormente o diagnóstico para o Covid-19, ainda que não haja menção alguma da suspeita do vírus na Declaração do Óbito, é possível que tal informação ingresse no assento por meio de uma averbação, de modo que o registro expresse a realidade" (CHOSSANI, 2020).

Com base nas informações de óbitos disponibilizadas pelo Registro Civil por mês de registro e mês de ocorrência, o presente estudo busca realizar uma comparação, por categoria de município, desses dois tipos de registro entre março e junho de 2020, no caso da Covid-19. 
A premissa é a de que, diante dos possíveis atrasos nos registros, os óbitos por mês de registro trazem informação temporalmente distinta daquela referente aos óbitos por mês de ocorrência. Além disso, propõe-se mensurar e avaliar a magnitude dessas diferenças quanto às datas.

\section{Métodos}

Os dados analisados de óbitos por mês de registro e mês de ocorrência foram obtidos no Portal da Transparência do Registro Civil (REGISTRO CIVIL, 2020), que mantém uma seção especial com registro dos óbitos relacionados à Covid-19, para municípios com mais de 50 casos suspeitos ou confirmados da doença. Os dados utilizados neste estudo referem-se ao período de 16 de março a 30 de junho de 2020 (Quadro 1).

\section{QUADRO 1}

Municípios com mais de 50 óbitos suspeitos ou confirmados de Covid-19, segundo categorias Brasil-março-junho 2020

\begin{tabular}{|lll|}
\hline $\begin{array}{l}\text { Categorias de } \\
\text { municípios }\end{array}$ & N (\%) & \multicolumn{1}{c|}{ Nome do município } \\
\hline Capitais & $22(19,6)$ & $\begin{array}{l}\text { Aracaju, Belém, Belo Horizonte, Boa Vista, Curitiba, Fortaleza, Goiânia, João } \\
\text { Pessoa, Macapá, Maceió, Manaus, Natal, Porto Alegre, Porto Velho, Recife, Rio } \\
\text { Branco, Rio de Janeiro, Salvador, São Luís, São Paulo, Teresina, Vitória }\end{array}$ \\
\hline $\begin{array}{l}\text { Municípios } \\
\text { pertencentes } \\
\text { a regiões } \\
\text { metropolitanas, } \\
\text { exceto capitais }\end{array}$ & $\begin{array}{l}\text { Barueri, Belford Roxo, Cabo de Santo Agostinho, Caieiras, Carapicuíba, } \\
\text { Cariacica, Caucaia, Cotia, Diadema, Duque de Caxias, Embu das Artes, Ferraz } \\
\text { de Vasconcelos, Francisco Morato, Franco da Rocha, Guarulhos, Itaboraí, }\end{array}$ \\
& $\begin{array}{l}\text { Itaguá, Itapecerica da Serra, Itapevi, Itaquaquecetuba, Jaboatão dos } \\
\text { Guararapes, Magé, Maracanau, Maricá, Mauá, Mesquita, Mogi das Cruzes, }\end{array}$ \\
& $\begin{array}{l}\text { Nilópolis, Nova lguaçu, Niterói, Olinda, Osasco, Paulista, Queimados, Santo } \\
\text { André, São Bernardo do Campo, São Caetano do Sul, São Gonçalo, São João } \\
\text { de Meriti, São José de Ribamar, Serra, Vila Velha }\end{array}$ \\
\hline $\begin{array}{l}\text { Municípios do } \\
\text { interior }\end{array}$ & $\begin{array}{l}\text { Ananindeua, Angra dos Reis, Arapiraca, Araruama, Bayeux, Cabo Frio, } \\
\text { Cachoeiro de Itapemirim, Campina Grande, Campinas, Campos dos } \\
\text { Goytacazes, Caruaru, Cascavel, Cubatão, Feira de Santana, Gama, Governador } \\
\text { Valadares, Guará, Guarujá, Imperatriz, Indaiatuba, Juiz de Fora, Jundiaí, } \\
\text { Limeira, Londrina, Macaé, Marabá, Mossoró, Nova Friburgo, Núcleo } \\
\text { Bandeirantes, Paragominas, Parauapebas, Petrópolis, Piracicaba, Praia } \\
\text { Grande, Ribeirão Preto, Rio Verde, Santa Rita, Santarém, Santos, São José } \\
\text { do Rio Preto, São José dos Campos, São Vicente, Sorocaba, Taguatinga, } \\
\text { Teresópolis, Uberlândia, Vitória de Santo Antão, Volta Redonda. }\end{array}$ \\
\hline Total & 112 (100,0) \\
\hline
\end{tabular}

Fonte: Central de Informações do Registro Civil - CRC Nacional.

A comparação entre óbitos por mês de registro e de ocorrência se deu mês a mês, calculando-se a magnitude da diferença entre os dois tipos de informação, segundo a categoria do município - capital, região metropolitana e interior.

Foram construídos modelos de regressão linear simples entre óbitos por mês de registro (variável dependente) ao longo dos quatro meses (variável independente), para se obter o crescimento médio mensal. De fato, utilizou-se a variável dependente na sua forma centralizada (subtraindo a média da variável original) em vez da variável original, tendo em vista que existem diferenças nos número de dias nos meses e o registro mensal poderia ser afetado com o acréscimo de mais um dia. Esses modelos foram construídos 
por categoria de município (três modelos: capital, região metropolitana, exceto capital, e interior). Em seguida, repetiu-se esse procedimento para os óbitos por mês de ocorrência e categoria de município, tendo sido elaborados, portanto, mais três modelos. Após suas estimações, eles foram comparados dois a dois, por tipo de município, e dispostos em três gráficos distintos, com a respectiva reta de ajuste linear.

Além dos gráficos, os parâmetros do modelo de regressão foram organizados em tabela. Investigou-se se os parâmetros eram diferentes e, além disso, se os coeficientes angulares eram diferentes entre si (por categoria de município). 0 coeficiente de determinação $\left(R^{2}\right)$ indicou os modelos que mais se ajustaram ao pressuposto de linearidade na análise de tendência temporal.

Para digitação dos dados obtidos no Portal da Transparência e a construção dos gráficos, foi utilizado o Editor de Planilhas Microsoft Excel for Mac/2011 versão 14.0. Para construção da variável centralizada, elaboração dos modelos lineares e obtenção dos parâmetros, empregou-se o pacote estatístico Stata/SE for Mac versão 12.0 (StataCorp, 2011).

\section{Resultados}

Os dados indicam que, em março, por mês de registro, havia 253 óbitos por Covid-19 nas capitais, 44 nos municípios das regiões metropolitanas (exceto capitais) e 26 nos municípios do interior do Brasil (Tabela 1). Contudo, o número de óbitos por mês de ocorrência, em março, foi, de fato, muito maior do que aquele observado por mês de registro: 450 (77,9\% maior) nas capitais, 88 (100\% maior) nos municípios pertencentes a regiões metropolitanas, exceto capitais, e 52 (100\% maior) no interior (Tabela 1). Em comparação a março, no período de abril a junho houve um aumento expressivo de óbitos tanto por mês de ocorrência quanto por mês de registro. Entretanto, dentre os meses analisados, apenas em junho o número de óbitos por mês de registro foi superior àquele por mês de ocorrência, para as três categorias de municípios, sendo que nos demais meses da série, o número de óbitos por mês de registro foi sempre inferior ao de óbitos por mês de ocorrência.

Os totais observados para o período analisado, por categoria de município (Tabela 1), sugerem que, embora as análises mês a mês apresentem diferenças importantes, ao longo do tempo os registros se adequam com o aperfeiçoamento mensal das informações e as diferenças se mostram inferiores a $1 \%$.

0 Gráfico 1 indica esse panorama por categoria de município. Em geral, se observadas as tendências de março a junho de óbitos ocorridos para os óbitos registrados nos respectivos meses, a pandemia estaria dando sinais de arrefecimento. Todavia, chamam a atenção as inclinações entre maio e junho, por parecerem substancialmente maiores para os óbitos por mês de registro comparativamente àqueles por mês de ocorrência. Tal situação é especialmente preocupante no caso dos municípios do interior, onde se percebe claramente um crescimento do número de óbitos por mês de registro (entre maio e junho), e não um início de platô, como indica o número de óbitos por mês de ocorrência (entre os mesmos meses). 


\section{GRÁFICO 1}

Número de óbitos por Covid-19 em municípios com mais de 50 casos suspeitos ou confirmados, por mês de ocorrência e de registro, segundo categorias de município Brasil - março-junho de 2020

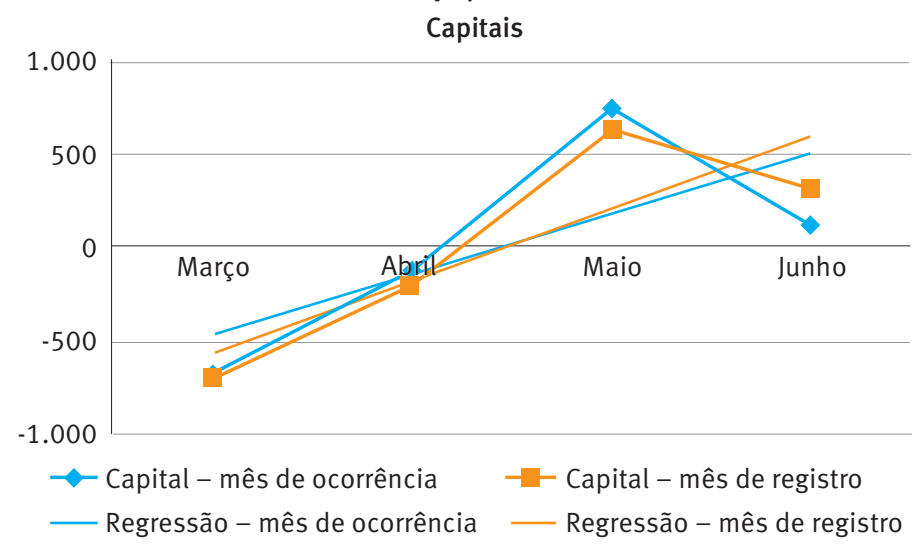

Municípios das regiões metropolitanas (exceto capitais)

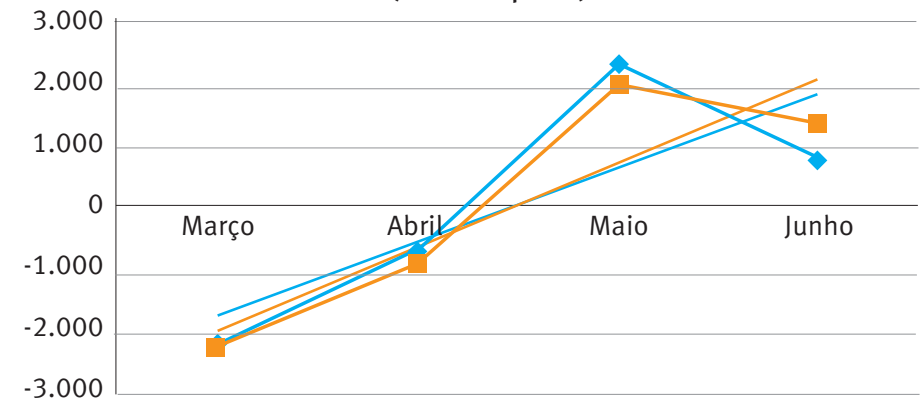

$\neg$ Região metrop. - mês de ocorrência - - Região metrop. - mês de registro

— Regressão - mês de registro _ _ Regressão - mês de ocorrência

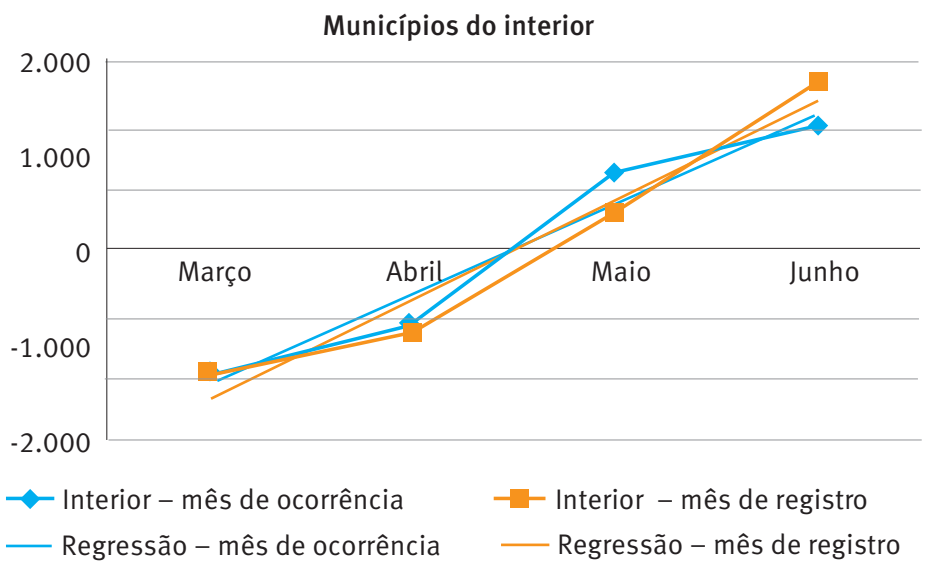

Fonte: Central de Informações do Registro Civil - CRC Nacional. 
TABELA 1

Número de óbitos por Covid-19 em municípios com mais de 50 casos suspeitos ou confirmados, por mês de ocorrência e de registrosegundo categorias de município Brasil - março-junho de 2020

\begin{tabular}{lrrr}
\hline \multicolumn{1}{c}{ Categorias de município } & $\begin{array}{c}\text { Mês de } \\
\text { ocorrência }\end{array}$ & $\begin{array}{c}\text { Mês de } \\
\text { registro }\end{array}$ & $\begin{array}{c}\text { Mês de ocorrência - mês de registro } \\
\text { (\% em relação ao mês de registro) }\end{array}$ \\
\hline Capital & 450 & 253 & $197(77,9)$ \\
Março & 6.107 & 5.273 & $834(15,8)$ \\
Abril & 14.680 & 13.735 & $945(6,9)$ \\
Maio & 8.517 & 10.485 & $-1.968(-18,8)$ \\
Junho & 29.754 & 29.746 & $\mathbf{8}(0,03)$ \\
Total & & & $44(100,0)$ \\
Região metropolitana, exceto capital & 88 & 44 & $263(19,1)$ \\
Março & 1.643 & 1.380 & $299(7,0)$ \\
Abril & 4.583 & 4.284 & $-572(-15,8)$ \\
Maio & 3.057 & 3.629 & $34(0,4)$ \\
Junho & 9.371 & 9.337 & $26(100,0)$ \\
Total & & & $69(14,6)$ \\
\hline Interior & 52 & 26 & $399(22,8)$ \\
Março & 542 & 473 & $-455(-14,7)$ \\
Abril & 2.149 & 1.750 & $39(0,7)$ \\
Maio & 2.648 & 3.103 & \\
Junho & 5.391 & 5.352 & \\
Total & & & \\
\hline
\end{tabular}

Fonte: Central de Informações do Registro Civil - CRC Nacional.

A Tabela 2 apresenta os resultados para as regressões lineares simples, que indicam sustentadamente coeficientes angulares mais elevados na sequência dos óbitos registrados frente aos ocorridos para as três categorias de municípios estudados. Por exemplo, no caso das capitais, na sequência de óbitos por mês de ocorrência, observa-se um acréscimo médio de 3.277 óbitos de um mês para o outro; no caso daqueles por mês de registro, esse aumento correspondeu a 3.916 óbitos. 0 mesmo comportamento é observado para o conjunto das regiões metropolitanas exceto capitais e para os municípios do interior. Nota-se, ainda, que as sequências de óbitos por mês de registro (coeficientes de determinação superiores) se adequaram melhor ao modelo linear, contudo, no caso do interior, a diferença foi mínima e ambos os coeficientes foram superiores a 0,90 .

TABELA 2

Coeficientes dos modelos de regressão linear dos óbitos por Covid-19 em municípios com mais de 50 casos suspeitos ou confirmados, por mês de ocorrência e de registro, segundo categorias de município (modelos para capital, região metropolitana exceto capital e interior) Brasil - março-junho de 2020

\begin{tabular}{|c|c|c|c|c|c|c|}
\hline \multirow{2}{*}{$\begin{array}{l}\text { Categorias de } \\
\text { município }\end{array}$} & \multicolumn{3}{|c|}{ Mês de ocorrência } & \multicolumn{3}{|c|}{ Mês de registro } \\
\hline & Intercepto & Angular & $\mathrm{R} 2$ & Intercepto & Angular & $\mathrm{R} 2$ \\
\hline Capital & -8.194 & 3.277 & 0,52 & -9.790 & 3.916 & 0,73 \\
\hline $\begin{array}{l}\text { Região metropolitana, } \\
\text { exceto capital }\end{array}$ & -2.962 & 1.185 & 0,63 & -3.415 & 1.366 & 0,80 \\
\hline Interior & -2.349 & 940 & 0,95 & -2.627 & 1.051 & 0,96 \\
\hline
\end{tabular}

Fonte: Central de Informações do Registro Civil - CRC Nacional. 


\section{Discussão}

O comportamento distinto das séries de óbitos por mês de ocorrência e de registro pode ter sido observado por alguns prováveis motivos. Em primeiro lugar, pode ter havido redistribuição dos óbitos registrados nos meses subsequentes, mas que, de fato, ocorreram em março. Em segundo lugar, pode ter ocorrido mudança na causa do óbito de alguma outra causa para Covid-19, após correção no Sistema de Informação de Mortalidade (SIM). Em terceiro lugar, diferentes municípios e unidades da federação reportam dados em velocidades distintas. A situação observada em junho, quando se nota que os óbitos por mês de registro, nas três classificações de municípios, foram superiores àqueles por mês de ocorrência, pode se dever ao fato de que nem todos os óbitos cujo mês de registro é junho referem-se a óbitos ocorridos em junho e, provavelmente, aqueles ocorridos em junho serão registrados posteriormente, assim como se observou para meses precedentes. Essa hipótese é bastante razoável, tendo em vista que os totais para os quatro meses são bastante similares, sugerindo que o diagnóstico e a qualidade das informações dos registros são aprimorados constantemente. Assim, fica claro que parte importante dos óbitos ocorridos em junho serão incorporados em estatísticas por mês de ocorrência em julho somente. Cabe ressaltar que o Brasil não é o único país que apresenta esses diferenciais entre óbitos por mês de registro e de ocorrência. Por exemplo, o Centers for Disease Control and Prevention (CDC) dos Estados Unidos e o Office of National Statistics da Inglaterra e País de Gales possuem uma página dedicada a estes dados durante a pandemia, os quais denominam de provisórios (CDC, 2020; ONS, 2020).

Quanto aos resultados por tipo de município, nota-se que a maior diferença entre óbitos por mês de ocorrência e de registro foi observada para os municípios do interior, o que é consistente com o alastramento da epidemia rumo ao interior iniciada naquele mês. Além disso, o coeficiente de determinação menos próximo de um $(1,0)$ indica menor aderência, ao modelo linear, das capitais e dos municípios de regiões metropolitanas. De fato, essa análise coincide com a impressão visual que indica que, para o interior, a relação é praticamente linear, mas para os outros dois grupos há um aumento curvilíneo e, depois, um declive.

\section{Conclusões e próximos passos a partir desta pesquisa preliminar}

Em epidemias e pandemias, como a de Covid-19, dados confiáveis e oportunos sobre a causa da morte são essenciais para definir medidas de controle da propagação da doença e gerenciar melhor os serviços de saúde (FRANÇA et al., 2020). A contabilização correta e oportuna de cada óbito é de extrema importância nesses contextos. Por exemplo, estima-se que, quando uma única morte ocorre em determinada localidade, centenas de casos, ou até mesmo milhares, provavelmente estariam presentes 
na população (JOMBART et al., 2020). Daí a importância do monitoramento adequado e correto dos óbitos. Recomenda-se, como próximos passos, o contínuo monitoramento dos diferenciais entre óbitos por mês de ocorrência e de registro até o final da pandemia de Covid-19.

Finalmente, seria de grande utilidade tanto aos demógrafos quanto aos estudiosos da área de saúde e da vigilância, no que se refere à Covid-19, identificar em que medida as tendências apresentadas destoam do que seria esperado para mortes em geral no Brasil, o que não foi ainda possível realizar com os dados disponíveis, tendo em vista haver informações do Registro Civil apenas para causas respiratórias e algumas cardiovasculares (recentemente incluídas no Portal da Transparência). Estudo do Reino Unido indica que óbitos por causas naturais apresentam diferenças menores do que óbitos por causas externas, contudo, não se sabe como seria no caso de uma doença que atinge todo o mundo (ONS, 2018). Ter indícios do que se pode esperar das estatísticas gerais de mortalidade por mês de ocorrência e por mês de registro, estratificando por grupos mais vulneráveis à Covid-19, por sexo e faixa etária, por exemplo, pode ser uma contribuição valiosa da demografia ao planejamento das ações de saúde, prevenindo novos óbitos pela doença.

\section{Referências}

CDC - Centers for Disease Control and Prevention. Daily updates of totals by week and state: provisional death counts for coronavirus disease 2019 (COVID-19). 2020. Disponível em: https:// www.cdc.gov/nchs/nvss/vsrr/covid19/index.htm. Acesso em: 19 jul. 2020.

CHOSSANI, F. W. O registro do óbito em tempos de Covid-19. São Paulo: Associação dos Registradores de Pessoas Naturais do Estado de São Paulo, 2020. Disponível em: http://www. arpensp.org.br/index.php?pG=X19leGlizV9ub3RpY2lhcw==\&in=OTQzNDc=. Acesso em: 15 jul. 2020.

FERNANDES, J. L. Covid-19 no Brasil: aprendendo a andar no escuro sem deixar nada para trás. Arquivos Brasileiros de Cardiologia, v. 114, n. 6, p. 988-991, jun. 2020. Disponível em: http:// publicacoes.cardiol.br/portal/abc/portugues/2020/v11406/pdf/11406001.pdf. Acesso em: 17 jul. 2020.

FRANÇA, E. B. et al. Deaths due to Covid-19 in Brazil: how many are there and which are being identified? Revista Brasileira de Epidemiologia, v. 23, 2020. Disponivel em: https://www.scielo. br/pdf/rbepid/v23/1980-5497-rbepid-23-e200053.pdf. Acesso em: 19 jul. 2020.

GAGLIARD, A. R.; MROZ, D. S.; MARCONI, E. L.; MUNNO, K.Z. O registro de óbito e sua retificação em virtude do Covid-19. São Paulo: Associação dos Registradores de Pessoas Naturais do Estado de São Paulo, 2020. Disponível em: https://infographya.com/files/Artigo_ArpenSP_ Retificac\%CC\%A7ao_e_COVID-19_versa\%CC\%830_final.pdf. Acesso em: 19 jul. 2020.

IBGE - Instituto Brasileiro de Geografia e Estatística. Pesquisa do Registro Civil do IBGE. Disponivel em: http://www.registrocivil.ibge.gov.br/. Acesso em: 19 jul. 2020.

JOMBART, T. et al. Inferring the number of COVID-19 cases from recently reported deaths. medRxiv, 2020. Disponível em: https://www.medrxiv.org/content/10.1101/2020.03.10.20033761v1. Acesso em: 19 jul. 2020. 
MACHADO, C. O Registro Civil frente à pandemia do Covid-19: recomendações internacionais e a resposta brasileira. Consultor Jurídico, 2020. Disponível em: https://www.conjur.com.br/dl/ registro-civil-versao-final-lieg.pdf. Acesso em: 19 jul. 2020.

REGISTRO CIVIL. Óbitos em cidades com mais de 50 casos suspeitos ou confirmados de Covid-19. Portal da Transparência, 2020. Disponível em: https://transparencia.registrocivil.org.br/especialcovid. Acesso em: 02 jul. 2020.

ONS - Office of National Statistics. Deaths registered weekly in England and Wales, provisional. 2020. Disponível em: https:/www.ons.gov.uk/peoplepopulationandcommunity/birthsdeathsandmarriages/ deaths/datasets/weeklyprovisionalfiguresondeathsregisteredinenglandandwales. Acesso em: 19 jul. 2020.

ONS - Office of National Statistics. Impact of registration delays on mortality statistics in England and Wales: 2018. 2020. Disponível em: https://www.ons.gov.uk/peoplepopulationandcommunity/ birthsdeathsandmarriages/deaths/articles/impactofregistrationdelaysonmortalitystatisticsine nglandandwales/2018\#the-delay-between-death-occurrence-and-registration-has-increasedin-2018. Acesso em: 24 ago. 2020.

STATACORP. Stata 12 Base Reference Manual. College Station, TX: Stata Press, 2011.

UNITED NATIONS. Maintaining Civil Registration and Vital Statistics during the COVID-19. 2020. Disponível em: https://unstats.un.org/legal-identity-agenda/documents/COVID-19-Guidelines. pdf. Acesso em: 19 jul. 2020.

XIE X. et al. Chest CT for typical 2019-nCoV pneumonia: relationship to negative RT-PCR testing. Radiology, 2020. Disponível em: https://doi.org/10.1148/radiol.2020200343. Acesso em: 20 jul. 2020.

\section{Sobre as autoras}

Claudia Cristina de Aguiar Pereira é doutora em Population Health (University of Wisconsin, Madison) e com pós-doutorado na Johns Hopkins Bloomberg School of Public Health. Pesquisadora do Departamento de Administração e Planejamento em Saúde da Escola Nacional de Saúde Pública Sergio Arouca (Ensp) da Fundação Oswaldo Cruz (Fiocruz).

Amanda Batista Marcelino é graduada em administração pela Pontifícia Universidade Católica de Minas Gerais (PUC Minas). Colaboradora do Núcleo Promoção de Saúde e Paz da Faculdade de Medicina da Universidade Federal de Minas Gerais (UFMG).

Carla Jorge Machado é doutora em Demografia pela Johns Hopkins University. Professora do Departamento de Medicina Preventiva e Social da Faculdade de Medicina da Universidade Federal de Minas Gerais (UFMG).

\section{Endereço para correspondência}

\section{Claudia Cristina de Aguiar Pereira}

Fundação Oswaldo Cruz, Escola Nacional de Saúde Pública

Rua Leopoldo Bulhões, $1480,7^{\circ}$ andar, Manguinhos

21041-210 - Rio de Janeiro-RJ, Brasil - caixa-postal: 22900993

\section{Amanda Batista Marcelino}

Universidade Federal de Minas Gerais, Faculdade de Medicina

Av. Professor Alfredo Balena, 190, $8^{\circ}$ andar, Centro

30130-100 - Belo Horizonte-MG, Brasil 


\author{
Carla Jorge Machado \\ Universidade Federal de Minas Gerais, Faculdade de Medicina \\ Av. Professor Alfredo Balena, 190, $8^{\circ}$ andar, Centro \\ 30130-100 - Belo Horizonte-MG, Brasil
}

\begin{abstract}
The Civil Registry in the first four months of the Covid-19 pandemic in Brazil: a comparative study between deaths by month of occurrence and by month of registration
\end{abstract}

The Civil Registry provides information on demographic studies on mortality, fertility and nuptiality. Questions have been raised about death registration when the causes of death are related to Covid-19. Notification may be delayed. Based on information provided by the Civil Registry of deaths, stratified by two groups - date of registration and date of occurrence - by Covid-19, this study compares these groups by city categories (capital, metropolitan region, interior), over March, April, May and June 2020, occurring in the first four months of the pandemic in Brazil. The magnitude and meaning of the differences in death records between the two groups were assessed. Graphs and linear regression analyses were used for comparisons. The findings indicate a different behavior of deaths per month of registration compared to deaths per month of occurrence between March and June. It is important to identify such a difference in behavior since, for the very short-term monitoring of the pandemic, deaths from registry data anticipate deaths whose records will still be adequately available by occurrence data. Such variation occurs mainly due to the systematic correction and updating of the information. As for the city categories, the biggest difference between deaths by month of occurrence and registration was observed for the municipalities in the interior, in line with the spread of the epidemic towards the interior in the month of May.

Keywords: Coronavirus infections. Mortality. Registries.

\title{
Resumen
}

El Registro Civil en los primeros cuatro meses de la pandemia de covid-19 en Brasil: un estudio comparativo entre muertes por mes de ocurrencia y por mes de registro

El Registro Civil proporciona información sobre estudios demográficos en mortalidad, fecundidad y nupcialidad. Se han planteado preguntas sobre el registro de muertes cuando las causas de muerte son por covid-19, ya que la notificación puede producirse tarde. Con base en la información proporcionada por el Registro Civil de Defunciones, desglosada en dos grupos -fecha de inscripción y fecha de ocurrencia- por covid-19, este estudio los compara por tipo de municipio (capital, región metropolitana, interior), sobre los meses de marzo, abril, mayo y junio de 2020, corresponden a primeros cuatro meses de la pandemia en Brasil. Se evalúa la magnitud y el significado de las diferencias en los registros de defunción entre los dos grupos. Para las comparaciones se utilizaron gráficos y análisis de regresión lineal. Los hallazgos indican un comportamiento diferente de las muertes por mes de registro en comparación con las muertes por mes de ocurrencia entre marzo y junio. Es importante identificar tal diferencia en el comportamiento ya que, para el monitoreo a muy corto plazo de la pandemia, las muertes a partir de los datos del registro anticipan muertes cuyos registros aún estarán disponibles 
correctamente mediante los datos de ocurrencia. Se concluye que esta variación se produce principalmente por la corrección y actualización sistemática de la información. En cuanto al tipo de municipio, la mayor diferencia entre defunciones por mes de ocurrencia y de registro se registró para los municipios del interior, en línea con la propagación de la epidemia hacia el interior, en mayo.

Palabras clave: Infecciones por coronavirus. Mortalidad. Sistema de registro

Recebido para publicação em 21/07/2020

Aceito para publicação em 02/09/2020 